\title{
Modeling and analysis of grinding force in ultrasonic honing considering the scale effect
}

\author{
Ye Linzheng , Zhu Xijing \\ Key Laboratory for AMT of Shanxi, North University of China, Taiyuan 030051, China
}

\begin{abstract}
To research the power ultrasonic honing mechanism at the micro scale, the scale effect is considered and the strain gradient plasticity theory based on the dislocation mechanism (MSG) is applied to establish the model of a whetstone grinding force, and the simulation analysis is conducted. Results show: the grinding force will increase when the scale effect is considered; the main influence parameter is honing depth on the grinding force; the grinding force increases nonlinearly with the continued reduce of honing depth after which decreases to $1.4 \mu \mathrm{m}$, which decreases slightly with the increase of the relative velocity of a whetstone. So the material becomes difficult to remove at the micro scale.
\end{abstract}

\section{Introduction}

Power ultrasonic honing is a new type of precision machining method, with some advantages such as small grinding force, low honing temperature and high processing efficiency, which is widely used in cylinder block and cylinder liner processing of cars and tanks, and has a broad application prospect.[1] In recent years, many scientists have observed the scale effect of materials at the micro scale extensively from experiments, that is, when the characteristic length of the non-uniform plastic deformation of the material is at the micron level, the material behavior is significantly different from that at the macro scale. In 1994, Fleck et al. observed the dimensionless torsional stiffness of copper wire increased almost two times when its diameter reduced from $170 \mu \mathrm{m}$ to $12 \mu \mathrm{m}$ in thin copper wire torsion test.[2] In1998, A.G.Evans and Stolken observed that the bending strength of the beam increased significantly with the decrease of the thickness of the thin beam in thin beam bending experiment.[3] A series of micro indentation experiments also showed that the hardness of material increased with the indentation decreasing to the micron level.[4,5]

There are having been many discussions about the grinding force model at home and abroad, but not much introduction about the grinding force model of ultrasonic honing. Li lijun divided the grinding force into the cutting force and the friction force, and established the mathematical model of grinding force.[6] Xie guizhi et al. studied the critical conditions for the removal of plastic deformation and the removal of brittle fracture in the process of engineering ceramic grinding, and set up corresponding grinding force model respectively.[7] Liu zhen et al. established the grinding force model of ultrasonic honing considering the strain rate of the material.[8]

In summary, materials processed will inevitably show scale effect when the honing depth of ultrasonic honing reduces to micron level, but some related 
researches have not yet been seen. Based on the ultrasonic honing processing mechanism, applying the strain gradient plasticity theory based on the dislocation mechanism(MSG theory) and the scale effect of material is considered, establishing the grinding force model and conducting its numerical simulation, and analyzing the scale effect' influence on the grinding force in this paper.

\section{The mechanism of ultrasonic honing processing}

There are three forms of vibration applied in ultrasonic honing: axial vibration, radial vibration and torsional vibration. Axial ultrasonic vibration is chosen in this paper. At the same time of the whetstone doing reciprocating and axial rotation motions, the axial ultrasonic vibration applied makes sinusoidal speed attached to the whetstone synthesis speed. Assuming the applied form of vibration is $A \sin 2 \pi f t, A$ and $f$ represent the amplitude of axial vibration applied and the frequency of vibration respectively, the synthesis speed of the whetstone is:

$$
v_{\text {ea }}=\sqrt{v^{2}+\left(v_{\mathrm{a}}+v_{\mathrm{f}}\right)^{2}}
$$

Where, $v_{\text {ea }}, v, v_{\mathrm{a}}$ and $v_{\mathrm{f}}$ are the synthesis speed, reciprocating speed, rotation speed and ultrasonic vibration speed of the whetstone respectively.

\section{Scale effect of the power ultrasonic honing}

Materials' scale effect should be considered when the honing depth of ultrasonic honing is a few microns. With the honing depth decreases, the hardness and the specific grinding energy of the material processed will increase nonlinearly, which is the so-called scale effect. Applying the strain gradient plasticity theory based on the dislocation mechanism, the shear strength of the material can be expressed by the dislocation density.

$$
\tau=\alpha \mu b \sqrt{\rho}=\alpha \mu b \sqrt{\rho_{\mathrm{s}}+\rho_{\mathrm{G}}}
$$

Where $\tau$ is the shear strength; $\alpha$ is an empirical constant between 0.2 to 0.5 , here is $0.5 ; \mu$ represents the shear modulus; $b$ indicates the Burgers vector length, which is $0.29664 \mathrm{~nm}$ for the $45 \mathrm{steel} ; \rho, \rho_{\mathrm{s}}$ and
$\rho_{\mathrm{G}}$ are the total dislocation density, the statistics stored dislocation density and the geometrically necessary dislocation density, respectively. $\rho_{\mathrm{G}}$ is related to the strain gradient at the microscopic scale.

$$
\eta=\frac{1}{\bar{r}} \rho_{\mathrm{G}} b
$$

Where $\bar{r}$ is the Nye factor, which reflects the ratio of the average density of geometrically necessary dislocation and the geometrically necessary density in the most effective arrangement, is 2 for a face-centered cubic polycrystal and 3 for a face-centered cubic single crystal.

The tensile flow stress in the crystal is shown in the formula (4).

$$
\sigma=\bar{m} \alpha \mu b \sqrt{\rho_{\mathrm{s}}+\rho_{\mathrm{G}}}=\sigma_{\mathrm{ref}} \sqrt{f^{2}(\varepsilon)+l \eta}
$$

Where $\bar{m}$ is the Taylor factor, which is $\sqrt{3}$ or 3 for isotropic materials or anisotropic materials, since the model of the grinding force is studied from the microscopic view in this paper, $\bar{m}=3 ; \sigma_{\text {ref }}$ is the uniaxial tension reference stress; $l$ is the characteristic length of materials proposed by Fleck.

$$
l=\bar{r}\left(\frac{\bar{m} \alpha \mu}{\sigma_{\mathrm{ref}}}\right)^{2} b
$$

Nix and Gao developed a kind of dislocation model to estimate the geometrically necessary dislocation density under a conical indenter, and combined (4) the relationship between indentation hardness and indentation depth can be obtained.

$$
\frac{H}{H_{0}}=\sqrt{1+\frac{h^{*}}{h}}
$$

Where $H_{0}$ is the indentation hardness without scale effect, which is $1.7052 \mathrm{Mp}$ for $45 \mathrm{steel} H$ is the hardness when the indentation depth is $h ; h^{*}$ is a parameter of the length dimension, which can be expressed in the formula (7).

$$
h^{*}=\frac{81}{2} b \alpha^{2} \tan ^{2} \gamma\left(\frac{\mu}{H_{0}}\right)^{2}
$$

Where $\gamma$ indicates the angle between the conical indenter surface and the surface of the material pressed. For standard Vickers indenter, the half angle of 
indenter tip is $68^{\circ}$, so $\gamma$ is $22^{\circ}$.

\section{Whetstone grinding force model}

\subsection{Single grain grinding force model}

The grinding force consists of deformation force by cutting and friction, for a single grain:

$$
\left\{\begin{array}{l}
F_{\mathrm{gN}}=F_{\mathrm{gNc}}+F_{\mathrm{gNs}} \\
F_{\mathrm{gT}}=F_{\mathrm{gTc}}+F_{\mathrm{gTs}}
\end{array}\right.
$$

Where g represents a single grain, $\mathrm{N}$ and $\mathrm{T}$ indicate normal force and tangential force respectively, $\mathrm{c}$ and $\mathrm{s}$ indicate deformation force by cutting and friction respectively.

An abrasive on the whetstone is regarded as the Vickers indenter, as the following relationship:

$$
P=\xi H R^{2}
$$

Where $P$ represents the load imposed on a single abrasive, $\xi$ is the indenter geometry factor, which is 2 for the Vickers indenter, $H$ is the hardness of the material, $R$ indicates the micro indentation size, and $R=a_{\mathrm{p}} \tan \lambda, a_{\mathrm{p}}$ is the indentation depth, $\lambda$, the half angle of indenter tip, is $68^{\circ}$.

Abrasive may not always contact with the workpiece due to the fact that the whetstone grain is not same with the standard Vickers indenter completely and the empty cutting phenomenon in ultrasonic honing, so a dimensionless coefficient $\eta_{0}\left(0<\eta_{0}<1\right)$ is introduced, according to the specific processing environment and experimental experience, is taken 0.5 , combined with (6) the normal cutting deformation force can be obtained.

$$
F_{\mathrm{gNc}}=\eta_{0} \xi H_{0} a_{\mathrm{p}}^{2} \tan ^{2} \lambda \sqrt{1+h^{*} / a_{\mathrm{p}}}
$$

The tangential cutting force can be obtained according to the force analysis of a single grain and the geometrical conditions.

$$
F_{\mathrm{gTc}}=\frac{\pi}{4} \eta_{0} \xi H_{0} a_{\mathrm{p}}^{2} \tan \lambda \sqrt{1+h^{*} / a_{\mathrm{p}}}
$$

For a single grain, the grinding force owing to the friction is shown as follows:

$$
\left\{\begin{array}{c}
F_{\mathrm{gNs}}=S \bar{P} \\
F_{\mathrm{gTs}}=\bar{\mu} S \bar{P}
\end{array}\right.
$$

Where $S$ represents the real contact area of a single grain and workpiece, due to the empty cutting phenomenon in ultrasonic honing, $S=\beta \pi\left(a_{\mathrm{p}} \tan \theta+r_{0}\right) 2+(1-\beta) \pi r_{0}^{2}, \beta$ is the real contact area ratio of abrasive and workpiece, $0<\beta<0.5$ generally, which is taken 0.4 on the basis of past experience, $\theta$ is the half cone angle of abrasive and $r_{0}$ is grinding flat particle radius, $\bar{P}$ is the average pressure on a single grain, and $\bar{P}=\frac{P}{\pi R^{2}}=\frac{\eta_{0} \xi}{\pi} H_{0} \sqrt{1+\frac{h^{*}}{a_{\mathrm{p}}}}, \bar{\mu}$ is related with the whetstone speed, and $\mu=A+B e^{-60 C v_{\text {ea }}}, A$ is the static friction coefficient 0.1 between grain and workpiece, $B$ is sliding friction coefficient $0.08, C=0.014$.

Grinding force model of a single grain can be obtained from the above as shown in formula (13).

$$
\left\{\begin{array}{c}
F_{\mathrm{gN}}=\eta_{0} \xi H_{0} \sqrt{1+h^{*} / a_{\mathrm{p}}}\left[a_{\mathrm{p}}^{2} \tan ^{2} \lambda\right. \\
\left.+\beta\left(a_{\mathrm{p}} \tan \theta+r_{0}\right)^{2}+(1-\beta) r_{0}^{2}\right] \\
F_{\mathrm{gT}}=\eta_{0} \xi H_{0} \sqrt{1+h^{*} / a_{\mathrm{p}}}\left\{\frac{\pi}{4} a_{\mathrm{p}}{ }^{2} \tan \lambda+(A\right. \\
\left.\left.+B e^{-60 C_{\mathrm{ca}}}\right)\left[\beta\left(a_{\mathrm{p}} \tan \theta+r_{0}\right)^{2}+(1-\beta) r_{0}^{2}\right]\right\}
\end{array}\right.
$$

\subsection{A whetstone grinding force model}

The effective grain number on a whetstone can be estimated.

$$
N_{\mathrm{d}}=\frac{4 b l}{\pi d_{\text {mean }}^{2}} \cdot p
$$

Where $b$ and $l$ are the width and length of a whetstone respectively, $d_{\text {mean }}$ is grain mean diameter, $p$ indicates the percentage of abrasive scratching and cutting on a whetstone, some studies show that the grains numbers proportion which genuinely participating in scratching and cutting on the surface of the diamond whetstone were $3.84 \%$ and $0.52 \%$.

A whetstone grinding force model can be obtained by making a single grain force and the effective grain number on a whetstone multiply, as shown in formula (15). 


$$
\left\{\begin{array}{c}
F_{\mathrm{N}}=\eta_{0} \xi H_{0} \sqrt{1+h^{*} / a_{\mathrm{p}}} \\
{\left[a_{\mathrm{p}}^{2} \tan ^{2} \lambda+\beta\left(a_{\mathrm{p}} \tan \theta+r_{0}\right)^{2}\right.} \\
\left.+(1-\beta) r_{0}^{2}\right] \cdot N_{\mathrm{d}} \\
F_{\mathrm{T}}=\eta_{0} \xi H_{0} \sqrt{1+h^{*} / a_{\mathrm{p}}} \\
\left\{\frac{\pi}{4} a_{\mathrm{p}}^{2} \tan \lambda+\left(A+B e^{-60 C_{\mathrm{ea}}}\right)\right. \\
\left.\left[\beta\left(a_{\mathrm{p}} \tan \theta+r_{0}\right)^{2}+(1-\beta) r_{0}^{2}\right]\right\} \cdot N_{\mathrm{d}}
\end{array}\right.
$$

\section{Simulation analysis of grinding force model}

In order to find out the influence of the scale effect on ultrasonic honing intuitively, and analyse the degree and trend of change on grinding force, applying Matlab to doing simulation analysis of the grinding force model established. The material of a workpiece is taken the 45 steel, for the diamonds grain, the grain size was $150 \#$ and the concentration is $100 \%$, the half cone angle is $\pi / 4$, the grinding flat particle radius is $0.015 \mathrm{~mm}$, the structure size of a whetstone is $6 \times 6 \times 100 \mathrm{~mm}$, the simulation parameters of power ultrasonic honing is shown in table 1.

Table 1. Parameters of power ultrasonic honing

\begin{tabular}{|c|c|}
\hline rotation speed & $80 、 125 、 200 、 315 \mathrm{r} / \mathrm{min}$ \\
\hline reciprocating speed & $50 、 100 、 150 、 200 \mathrm{~mm} / \mathrm{s}$ \\
\hline Honing depth & $1 \sim 10 \mu \mathrm{m}$ \\
\hline Ultrasonic frequency & $18.6 \mathrm{KHz}$ \\
\hline Ultrasonic amplitude & $0.01 \mathrm{~mm}$ \\
\hline
\end{tabular}

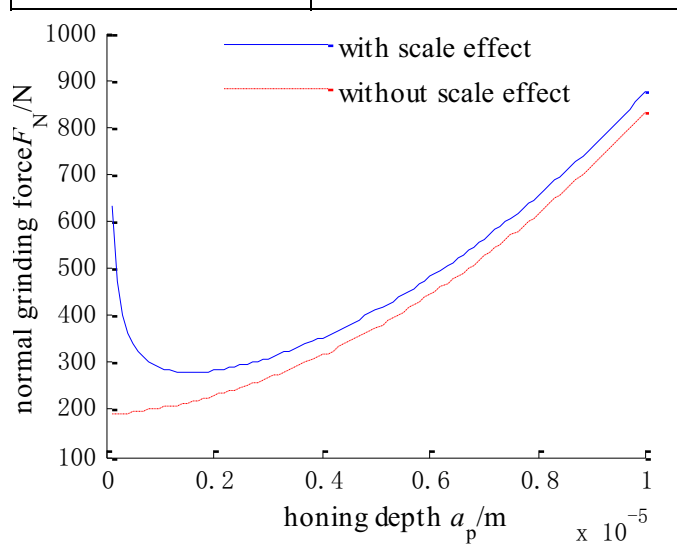

(a) $n=200 \mathrm{r} / \mathrm{min}, v_{\mathrm{a}}=100 \mathrm{~mm} / \mathrm{s}$

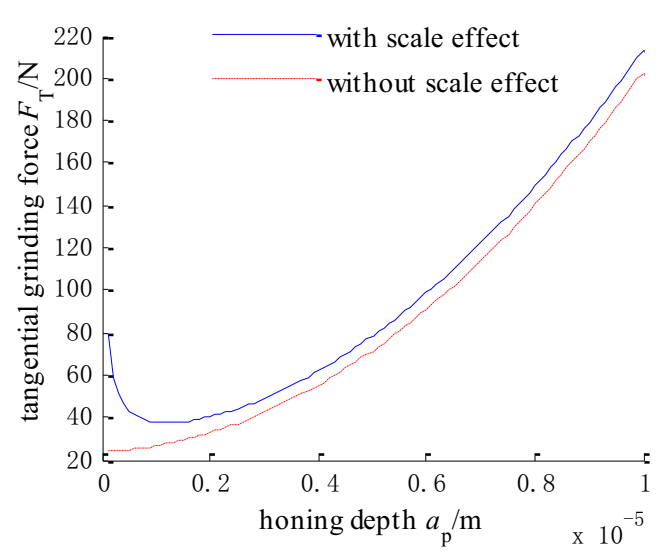

(b) $n=200 \mathrm{r} / \mathrm{min}, v_{\mathrm{a}}=100 \mathrm{~mm} / \mathrm{s}, t=1 / f$

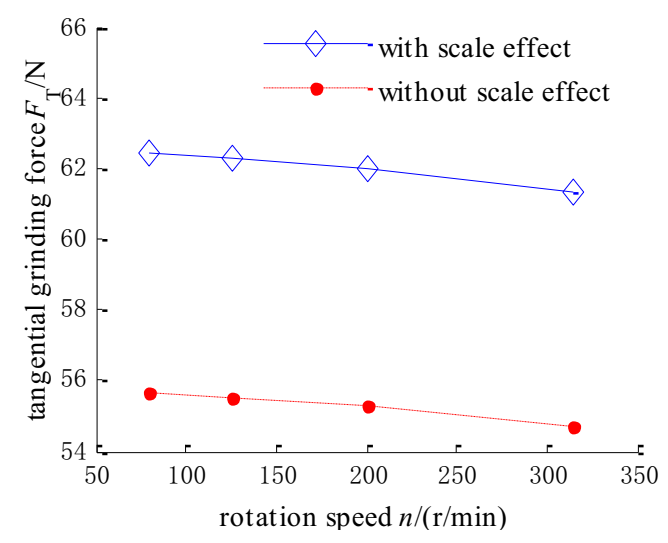

(c) $a_{\mathrm{p}}=4 \mu \mathrm{m}, v_{\mathrm{a}}=100 \mathrm{~mm} / \mathrm{s}, t=1 / \mathrm{f}$

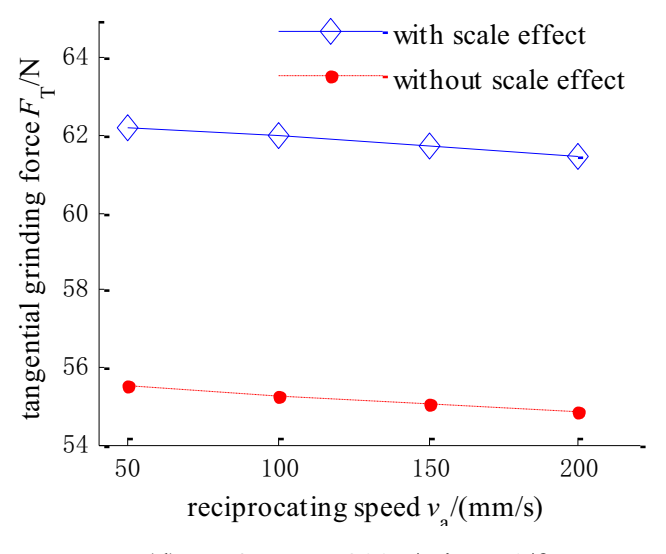

(d) $a_{\mathrm{p}}=4 \mu \mathrm{m}, n=200 \mathrm{r} / \mathrm{min}, t=1 / \mathrm{f}$

Figure 1. Curves of Grinding Force Change

Simulation results are shown in figure 1. As shown in (a) (b) (c) (d), honing depth is the main parameter influencing the grinding force, reciprocating and rotation speed is relatively small, compared with no considering the scale effect, the normal and tangential grinding force all increase under the influence of the scale effect. From (a) and (b) can see, the normal and tangential grinding force all change nonlinearly with the reduce of honing depth, which decrease with the decrease of honing depth, when the honing depth reduces to a certain value (about $1.4 \mu \mathrm{m}$ ), the grinding 
force reaches the minimum, and if honing depth continues to decrease, the grinding force will increase rapidly, so that material is difficult to remove. This is due to some changes of material characteristic at micro scale, the lattice dislocation needs to be fully considered, the hardness, shear strength and tensile flow stress of the material increase and the grinding force increases. When the honing depth is greater than the certain value, gradually tending to a macroscopic cutting, grinding force increases with the increase of honing depth. The grinding force reduces slightly with the increase of the reciprocating or rotation speed, because the increase of whetstone speed will reduce the dynamic friction coefficient so as to decrease the grinding force.

\section{Conclusions}

The scale effect should be considered when the honing depth of power ultrasonic depth is a few microns, which is essential for the microscopic mechanism of ultrasonic honing. The grinding force established is related with the microscopic parameters of the material processed, such as the Burgers vector length. When the scale effect is considered, the grinding force increased obviously, the normal and tangential grinding force will increase nonlinearly with the reduce of honing depth when the honing depth is less than $1.4 \mu \mathrm{m}$, and the grinding force decreases slightly with the increase of the relative velocity of a whetstone.

\section{References}

1. Zhu Xijing, Wang Jianqing, Cheng Quan, et al. Radial power ultrasonic vibration honing friction analysis[J]. Solid State Phenomena, 2011, 175: 183-186.

2. Fleck N A, Muller G M, Ashby M F, et al. Strain gradient plasticity: theory and experiment $[\mathrm{J}]$. Acta Metallurgica Et Materialia, 1994 42(2):475-487.

3. Stolken J S, Evans A G. A microbend test method for measuring the plasticity length scale [J]. Acta Materialia, 1998, 46(14):5109-5115.

4. Poole W. J., Ashby M. F., Fleck N. A.
Micro-hardness of annealed and work-hardened copper polycrystals[J]. Scripta Materialia, 1996, 34(4):559-564.

5. Mcelhaney K. W., Vlassak J. J., Nix W. D. Determination of indenter tip geometry and indentation contact area for depth-sensing indentation experiments [J]. Journal of Materials Research, 1998, 13(05):1300-1306.

6. Li Lijun, Fu Jiecai. A Study of Grinding Force Mathematical Model [J]. Journal of Hunan University, 1979, 3:44-55.

7. Xie Guizhi, Shang Zhentao, Sheng Xiaomin, et al. Grinding Force Modeling for High-speed Deep Grinding of Engineering Ceramics [J]. Journal of mechanical engineering. 2011, 47(11): 167-176.

8. Liu Zhen, Zhu Xijing, Wang Jianqing, et al. Modeling and Simulation of Grinding Force on Axial Power Ultrasonic Honing [J]. Mechanical Science and Technology for Aerospace Engineering. 2014, 33(6):858-86 